\title{
Chromoblastomycosis mimicking Squamous Cell Carcinoma: a case report
}

\author{
${ }^{1}$ Kayastha BMM, ${ }^{2}$ Shrestha $\mathrm{R},{ }^{2}$ Shrestha $\mathrm{P},{ }^{2}$ Karki A \\ ${ }^{1}$ Associate Professor and Senior Consultant Dermatologist \\ ${ }^{2}$ Residents \\ Department of Dermatology and STD \\ National Academy of Medical Sciences (NAMS), \\ Bir Hospital, Kathmandu, Nepal \\ Correspondence: bmkayastha@gmail.com
}

\section{Abstract}

Chromoblastomycosis (CM), a chronic subcutaneous mycosis, is caused by several dematiaceous fungi, the most common being Fonsecaea pedrosoi . It usually occurs in the lower extremities following traumatic implantation of the organisms. We are reporting a case of rapidly developing case of CM on the unilateral lower limb with a fungating mass like ulcerative lesion and hyperkeratotic warty growth. We could not explain the pattern of this rapid growth which seems to be one of the rare presentations. Scraping from a verrucous lesion in potassium hydroxide preparation revealed mycelia arising from sclerotic bodies The histopathology from the warty lesion showed granulomatous lesion without muriform or medlar bodies. The histopathology from the ulcerative lesion did not show any malignant changes. Our case responded very well to itraconazole. This case is presented here for the rapidity of growth and the development of a fungating mass and bleeding that simulated the behaviour of squamous cell carcinoma.

\section{Introduction}

Chromoblastomycosis (CM) is a chronic granulomatous mycotic infection of the skin and subcutaneous tissue caused by pigmented fungi, the most common being $F$. pedrosoi. It typically occurs on the exposed surfaces of the lower leg following traumatic implantation of the organisms. The lesions can involve other sites either by direct spread, autoinoculationor, or, by hematogenous spread. Treatment of CM is frequently difficult and unsatisfactory. We are reporting a case of CM with rapid evolution that responded very well to the treatment with itraconazole.

\section{Case Report}

A 50-year-old male agricultural worker of low socioeconomic status from Ramechhap district of Nepal, weighing $52 \mathrm{~kg}$, presented with intensely pruritic hyperkeratotic warty nodules involving the whole of left leg and dorsum of foot with a fungating ulcerated mass over lateral malleollus of the same leg for three months. On enquiry the patient did not recall any history of trauma. The lesion had started as a warty nodule on left midshin. As it was extremely pruritic he shaved it off with a blade only to reappear with multiple small satellite lesions around the original lesion. It grew in size rapidly rapidly in size to involve almost whole of the left leg below knee down to the anterior and lateral aspects of ankle and dorsal aspect of foot to the base of toes. On examination the lesions were discrete as well as confluent with islands of normal skin ; and, there was cauliflower- like fungating mass with oozing of sero- sanguinous and purulent foul smelling discharge with rolled out border on the lateral malleolar region with maggots wriggling within the lesion (Figures 1 $\& 2$ ). There was ipsilateral inguinal lymphadenopathy. Two lymph nodes of $2 \times 2 \mathrm{~cm}$ each were palpable which were non-tender,discrete, hard and fixed with the underlying structure. Systemic examination revealed no abnormality.

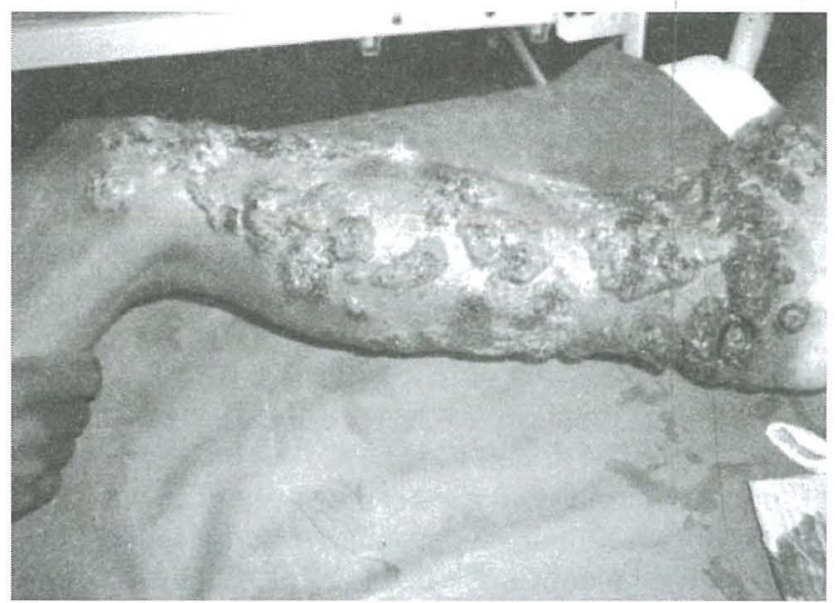

Fig.1. Irregular warty plaques and nodules involving whole of left leg and proximal part of dorsal foot. 


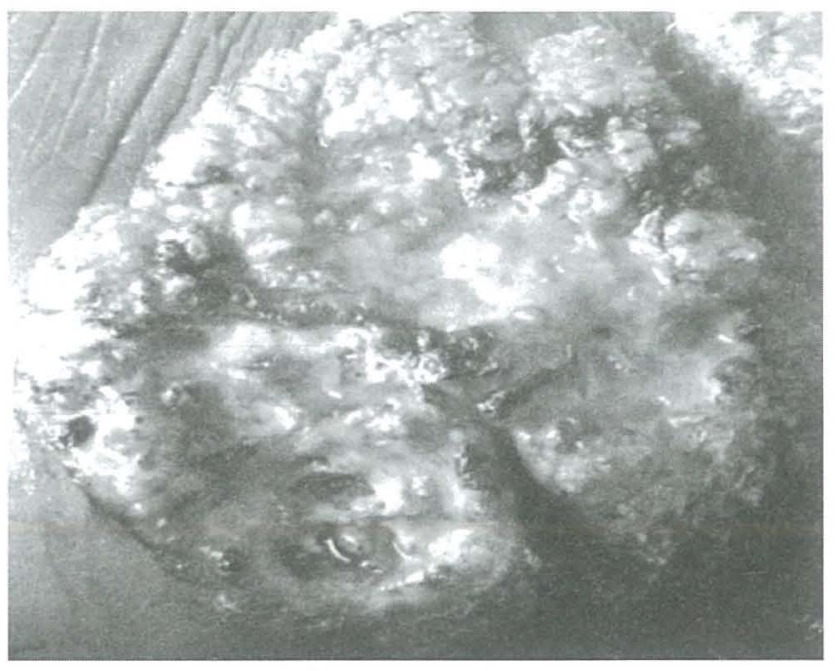

Fig. 2. A fungating ulcerated mass with maggots around left lateral malleolus.

We admitted the patient for further investigation and performed routine blood tests, gram stain showed gram positive coccobacilli and culture of the pus yielded mixed growth of organisms. Mantoux test and HIV antibody test were negative; chest $\mathrm{x}$-ray was normal.

Biopsy of the warty lesion showed well formed granuloma in the dermis, comprising of epithelioid cells, lymphocytes, Langhans giant cells without caseation necrosis with neutrophilic infiltration and formation of microabscesses. However no evidence of any fungal element was seen. PAS stain was also done but it didn't show fungal hyphae or spores. Biopsy from the fungating mass was negative for malignant cells. However the scraping from a verrucous lesion in potassium hydroxide preparation revealed mycelia arising from sclerotic bodies.

Based on the clinical features and positive $\mathrm{KOH}$ preparation we diagnosed this as a case of Chromoblastomycosis and started Itraconazole $100 \mathrm{mg}$ twice daily along with daily normal saline dressing after irrigation with hydrogen peroxide solution. About 2 dozen maggots were extracted from the ulcer. Cefadroxil $500 \mathrm{mg}$ twice daily was given for 7 days. There was dramatic improvement of the lesions within a week of starting itraconazole (Figure 3), and it continued to show improvement in the successive weeks with all the hyperkeratosis gone and erythematous plaque remaining (Figure 4). The patient continued to show improvement during the hospital stay and he was discharged with the advice to report if there was any complication or relapse. The patient has not turned up again until now.

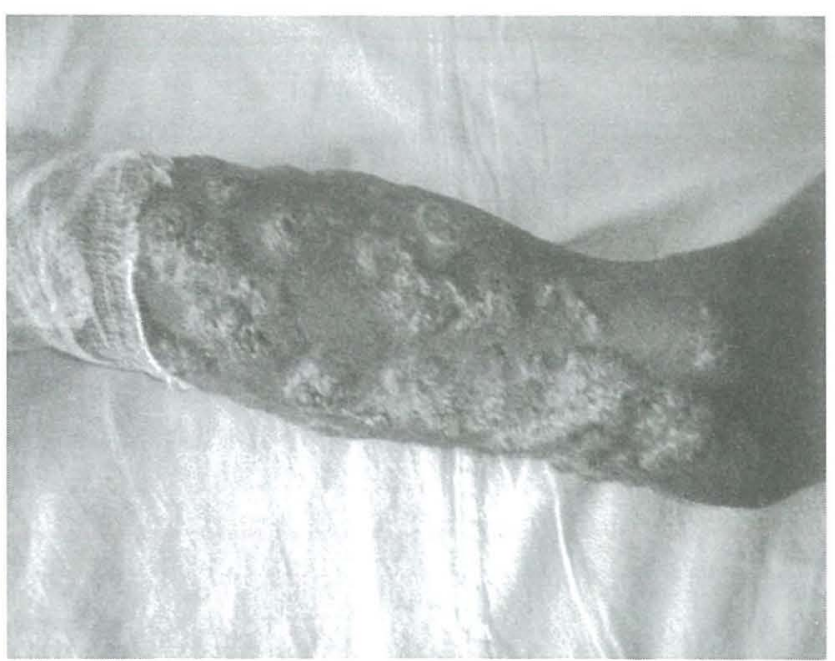

Fig. 3. After 1 week of itraconazole therapy.

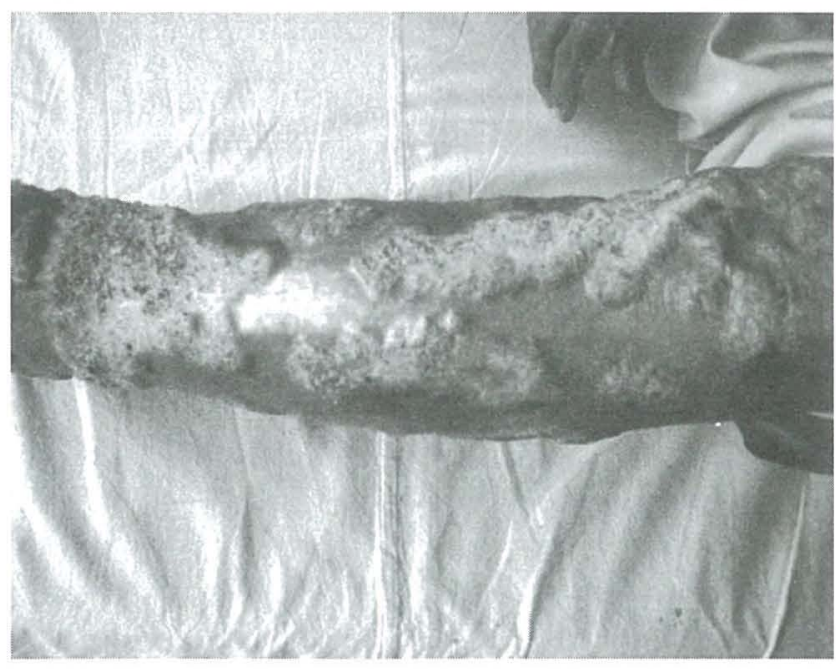

Fig. 4. After 4 weeks of treatment with itraconazole.

\section{Discussion}

Chromoblastomycosis(CM), also known as chromomycosis, verrucous dermatitis and dermatitis verrucosa chromoparasitaria, is a slowly progressive subcutaneous mycoses and is ubiquitous; however the highest incidence of chromoblastomycosis in the world is in the African country of Madagascar, ${ }^{1}$ and a large number of cases have been reported in Japan (Castro et al., 2006). Other major endemic areas include some islands of the Indian Ocean, South Africa, Asia, the Caribbean and Amazonian areas with the greater incidence in tropical and subtropical regions located between $30^{\circ} \mathrm{N}$ and $30^{\circ} \mathrm{S}$ (Brygoo, 1975). ${ }^{2}$ Brazil in South America is also one of the countries with highest incidence of $\mathrm{CM} .{ }^{3} \mathrm{CM}$ is attributed to several different dematiaceous fungi, including Fonsecaea pedrosoi, Phialophora verrucosa, Cladosporium carrionii, or Fonsecaea compacta, as well as a few other genera anecdotally found in the literature. ${ }^{4,5}$ These organisms are found in soil,wood and decaying plant material. CM is quite rare in Nepal, and can cause some diagnostic difficulties. A report of 2 cases of CM from Nepal was given by Agarwalla et al., in 2002. ${ }^{6}$ 
Approximately $70 \%$ of cases are seen in men. The explanation for this male predominance is not clear, but men are assumed to be more commonly involved in agricultural work and are more prone to inflict injuries on themselves, thereby causing self-inoculation, rather than women who are supposedly more dedicated to house jobs. The possible inhibitory effect of female hormones on the growth of fungi may partially explain the relatively low number of cases in females (Castro et al., 2006). In 1989, Tsuneto et al demonstrated a higher frequency of HLA-A29 in patients with $\mathrm{CM}$; this finding suggests that genetics might have an influence in the acquisition of the disease. ${ }^{7}$ The initial description of $\mathrm{CM}$ is credited to Alexandrino Pedroso in 1911 in Brazil. Now a days, the term CM is restricted to the cases in which sclerotic cells are present in tissue. Sclerotic cells, also known as Medlar bodies, are globeshaped, cigar-colored, thick-walled structures that are 4-12 $\mathrm{mm}$ in diameter. Medlar first described them in $1915 .^{8}$ These structures multiply by septation, and they induce a purulent and granulomatous inflammatory reaction in tissue. The exact pathogenesis of CM is unknown. Direct percutaneous inoculation, inhalation, and hematogenous dissemination have been implicated. The agents often gain entry into the human body by contact with wood splinters or thorns that is often not remembered or realized by the patient.. The primary lesion is thought to develop as a result of percutaneous traumatic inoculation. ${ }^{9}$ Rubin et al. have reported a case of CM that followed a well-defined episode of penetrating trauma. The causative organism, Fonsecaea pedrosi, was cultured from the patient's lesion and from the tree branch responsible for the trauma. This "natural" experiment supports the contention that one cause of CM is traumatic cutaneous implantation of the fungus. ${ }^{10}$ From the site of inoculation, the lesion usually restricts itself to cutaneous and subcutaneous tissue, especially in parts of the body not protected by clothing.

The lesions develop slowly at the site of implantation (only about $2 \mathrm{~mm}$ per year), producing a warty nodule, which tends to be limited to the skin and the subcutaneous tissue. Over the years, the nodular type of lesion normally develops into verrucous, pedunculated, cauliflowerlike florets, while the plaque type spreads peripherally, with an active, raised border, leaving a central healed area with atrophic and yellowish or ivory-colored scars. ${ }^{11}$ The disease tends to spread to neighboring healthy skin, forming plaques, which, at times, can involve a whole limb. When nodular lesions predominate over the plaques, the disease assumes a more typical cauliflower aspect. Both lymphatic dissemination and cutaneous dissemination have been described.
On the surface of both types of clinical variants, numerous black dots may be observed where the causative organisms are preferentially found. Hemopurulent material covering small ulcerations is commonly observed.Secondary infection with bacteria is common, giving the lesion a characteristic ill odor. Secondary infection is believed to be important in the genesis of lymph stasis and consequently of elephantiasis. In old cases, lesions in different stages of development can be found. Contiguous spread to the underlying bone may produce an osteolytic lesion. If not diagnosed earlier, CM has a chronic evolutional course that may cause several problems, such as difficulty in managing therapy because of the recrudescent character of the disease and poor quality of life and work incapacity to the patient. ${ }^{12}$ The most frequent complication is secondary bacterial infection, giving the lesion a characteristic ill odor. Secondary infection is believed to be important in the genesis of ulceration, lymph stasis and consequently of elephantiasis. Rarely, squamous cell carcinoma (SCC) develops within longstanding $\mathrm{CM}^{13}$ (14 cases are reported in the literature). ${ }^{14}$ Central nervous system invasion is possible and may be fatal. ${ }^{15}$

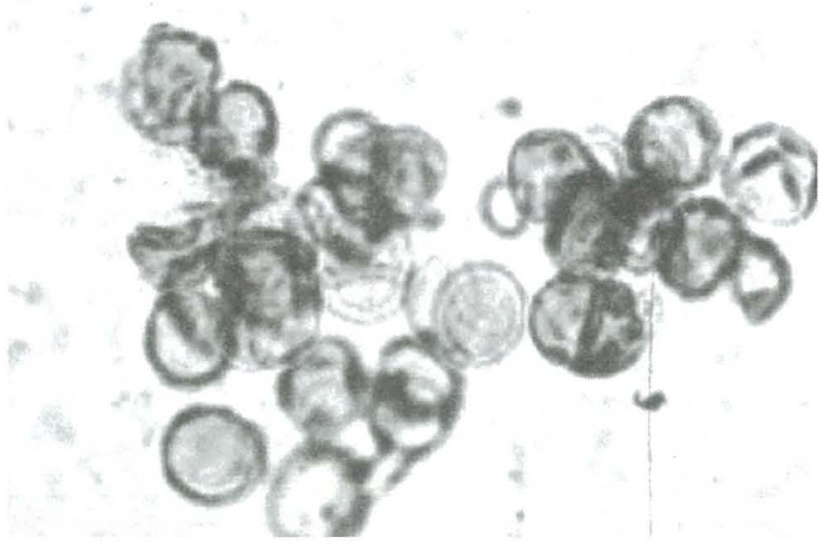

Fig.5. Sclerotic cells on a potassium hydroxide preparation.

Among the possible laboratory tests to be obtained, direct examination of $10 \%$ potassium hydroxide cleared lesion scrapings is by far the most useful. Typical, thick-walled, cigar-colored, sclerotic cells, also known as Medlar bodies, are readily seen (Figure 5).

In cutaneous lesions, the cigar-colored fungi are easily seen in hematoxylin and eosin-stained sections. No special stains are needed. The typical finding is a pseudoepitheliomatous hyperplasia of the epidermis with a diffuse, lymphomononuclear inflammatory infiltrate in the dermis. The tissue response to the fungus is typically mixed. True and pure abscesses or microabscesses, granulomas, granulomatous reactions, and abscesses surrounded by a granulomatous reaction with giant cells may 
be seen in the same section (Salfelder, 1990). Inside the giant cells, brown-colored, thick-walled fungal cells may be seen. These muriform fungal cells may be single, 2-celled, or multiple-celled; this feature is a result of multiplication by splitting rather than budding.

Mycologic tests are used to confirm the diagnosis. Culture in Sabouraud glucose agar media or Micosel agar allows isolation and identification of causal organisms within about 15 days. In 1999, Castro devised an elegant, clinically based, physician friendly index to stage chromoblastomycosis. The severity index is based on the extension of the diseased area, the number of lesions, the presence of complications (eg, lymphedema, ulceration, secondary infection), and the unresponsiveness to previous treatments. According to this scoring system, patients are classified as having mild (up to 3 points), moderate (4-6 points), or severe (7-10 points) disease.

\section{Scoring syst e m for staging chromoblastomycosis:}

- Area of lesions: Small lesions up to $25 \mathrm{~cm}^{2}$ are 1 point. Medium lesions larger than $25 \mathrm{~cm}^{2}$ and smaller than $100 \mathrm{~cm}^{2}$ are 2 points. Lesions larger than $100 \mathrm{~cm}^{2}$ are 3 points.

- Number of lesions: A single lesion is 1 point. One to 5 lesions is 2 points. More than 5 lesions or metastatic lesions is 3 points.

- Complications (1 point for each complication present): Lymphedema is 1 point. Ulceration is 1 point. Secondary infection is 1 point.

- Resistance to previous treatment or previous unsuccessful treatment is 1 point.

One of the most characteristic features of chromoblastomycosis is its refractoriness to treatment. Treatment of the disease presents poor effectiveness and serious side effects. ${ }^{16}$ Treatment is difficult and unsatisfactory too. ${ }^{17}$ Over the last decades, several different therapeutic schemes have been used, but most proved unsuccessful or of low efficacy. Chromoblastomycosis is a difficult condition to treat, especially if it is caused by Fonsecaea pedrosoi. Several therapeutic approaches have been used, including heat, surgery, cryotherapy, thiabendazole, amphotericin B combined with flucytosine, and azole derivatives, but their success has been modest. A 65\% response rate has been obtained with itraconazole given for periods of 6 to 19 months; in limited trials, saperconazole appears to be more effective and requires shorter treatment courses ${ }^{18}$ Surgical excision or electrodesiccation of lesions should be avoided because metastasis might ensue. To date, 2 therapeutic approaches are accepted as the best choices: oral itraconazole $100-200 \mathrm{mg} /$ day (as monotherapy ${ }^{19}$ or with oral 5-FC100-200 mg/day ${ }^{20}$ ) and cryosurgery with liquid nitrogen. In longstanding cases, removal of prominent cutaneous lesions by shave excision plus cryotherapy is also very helpful. Terbinafine $(250-500 \mathrm{mg} /$ day $)$ for up to 12 months achieved good results in 42 Malagasy patients suffering from chromoblastomycosis ${ }^{21}$ Amphotericin B $(1 \mathrm{mg} / \mathrm{kg} /$ day $)$ intravenously has been used in severe forms. Muhammed et al., reported a case of lymphangitic chromoblastomycosis responding well to itraconazole. ${ }^{22}$

Posaconazole, a new triazole derivative, has been experimentally used to treat chromoblastomycosis. Heat therapy is another treatment. Especially in Japan, the use of pocket warmers has proven successful in the treatment of a limited number of cases. Apparently, an increase in skin temperature somehow impairs fungal development. ${ }^{23}$ The prognosis tends to be good, especially for small and localized lesions. When the affected area is large, as in severe cases, cure is difficult, although control is easily achieved.Cicatricial and unaesthetic scars are the rule after the disease is eliminated (Castro et al., 2006).

\section{References:}

1. Esterre P, Andriantsimahavandy A, Ramarcel ER, pecarrere JL. Forty years of chromoblastomycosis in Madagascar: a review. Am J Trop Med Hyg 1996;55:45-47.

2. Brygoo ER, Destombes P: Epidemiologie de la chromoblastomycose humaine. Bull Inst Pasteur 1975; 74: 219-43.

3. Castro LGM, Schwartz RA Baran E.Chromoblastomycosis. > > Last Updated: September 25, 2006.

4. Maslin J, Morand JJ, Civatte M. Chromomycoses (chromoblastomycoses). Med Trop 2001;61:459-61. UI 11980389.

5. Rubin HA, Bruce S, Rosen T, McBride ME: Evidence for percutaneous inoculation as the mode of transmission for chromoblastomycosis. J Am Acad Dermatol 1991 Nov; 25(5 Pt 2): 951-4.

6. Agarwalla A,Khanal B, Garg VK, Agrawal S, Jacob M, Rani S, Deb M.

Chromoblastomycosis: report of two cases from Nepal. J Dermatol 2002;29(5):315-9,

7. Tsuneto LT, Arce-Gomez B, Petzl-Erler ML, Queiroz-Telles F: HLA-A29 and genetic susceptibility to chromoblastomycosis. J Med Vet Mycol 1989; 27(3): 181-5.

8. Medlar EM: A cutaneous infection caused by a new fungus Phialophora verrucosa with a study of the fungus. J Med Res 1915; 32: 507 22.

9. N Ezzine-Sebaï1 MD, R Benmously1 MD, B Fazaa1 MD E Chaker2 MD, R Zermani3 MD, 
and MR Kamoun1MD Chromomycosis arising in a Tunisian man.Dermatol Online J 2005; 11 (2): 14 .

10. ubin HA,ÊBruce S,ÊRosen T,ÊMcBride ME. Evidence for percutaneous inoculation as the mode of transmission for Chromoblastomycosis. J Am Acad Dermatol 1991, 25(5):951-4.

11.SchwartzRA, BaranE. Chromoblastomycosis.In: KimballAFB,; ButlerDF, CallenJP, Catherine QuirkC, Dirk M ElstonDM,eds. > > Last Updated: Feb 11, 2008.

12. Minotto R, Varejao BCD, MallmannÊLF, Albano E,ÊMaria I, ScrofernekerÊML. Chromoblastomycosis : A review of 100 cases in the state of Rio Grande do Sul, Brazil J Am Acad DermatolE Ê2001; $\hat{E} \hat{E} 44(4): \hat{E} 585-592$.

13. Chromoblastomycosis DermNet NZ.htm.Created 2003. Last updated 26 Dec 2006. (C) 2007.

14. Esterre P, Pecarrere JL, Raharisolo C, Huerre M. Squamous cell carcinoma arising from chromomycosis. Report of two cases. Ann Pathol 1999;19:516-20.

15. Artüz F, Alli N, Lenk N, Güngör E. Purple erythematous plaques on the face and left arm. Arch Dermatol 1997; 133:1027-1032. 16. SantosAL; PalmeiraÊVF; RozentalÊS; KneippLF; NimrichterÊL; AlvianoÊDS;
RodriguesÊML; AlvianoÊCS. Biology and pathogenesis of Fonsecaea pedrosoi, the major etiologic agent of chromoblastomycosis.. 17. TuffanelliLA,Milburn PBA. Treatment of Chromoblastomycosis. J Am Acad Dermatol 1990,$23 ; 4: 728-32$.

18. A Treatment of tropical mycoses. J Am Acad Dermatol 1994; 31:S91-102J .

19. Smith CH, Barker JN, Hay RJ. A case of chromomycosis responding to treatment with itraconazole. Int J Dermatol 1993;128:436-9.

20. Bolzinger T, Pradinaud R, Sainte-Marie D, Dupont B, Chwetzoff E. Traitement de quatre cas de chromomycose à Fonsecaea pedrosoi par l'association 5-fuorocytosine-itraconazole. Nouv Dermatol 1991;10:462-6.

21. Hay RJ. Therapeutic potential of terbinafine in subcutaneous and systemic mycoses. Br J Dermatol.1999;141 Suppl 56:36-40.

22. Muhammed K, Nandakumar G, Asokan KK, Vimi P. Lymphangitic chromoblastomycosis. Indian J Dermatol Venereol Leprol 2006;72:443-445.

23. Kinbara T, Fukushiro R, Eryu Y: Chromomycosis--report of two cases successfully treated with local heat therapy. Mykosen 1982 Dec; 25(12): 689-94. 